\title{
The interaction of target size and background pattern on perceived velocity during visual tracking
}

\author{
J. E. RAYMOND \\ University of Calgary, Calgary, Alberta, Canada
}

\begin{abstract}
The effect of target size and background contour on the perceived velocity of a visually tracked target was determined using a psychophysical comparison procedure. For targets viewed against a uniform field, an increase in target size produced a modest underestimation of target velocity. For small test targets, a background of contours moving in the same direction as target motion also produced an underestimation of target velocity. A background of contours moving in the direction opposite to that of target motion produced a small, but consistent, overestimation of target velocity. As target size increased, the underestimation effect seen with striped backgrounds moving in the same direction was not enhanced. The overestimation effect seen with a striped background moving in the opposite direction was nulled by an increase in target size.
\end{abstract}

Early studies by Brown (1931) reported that the perceived velocity of a smoothly moving target depended on the target's size and on the presence of surrounding contour. A large dot moving within a large rectangular frame was perceived to be moving more slowly than a small dot moving at the same speed within a smaller rectangular frame. The separate effects of target size and extent of stationary background contour were not assessed. Brown's effect, known as velocity transposition, has been used to explain automobile accidents that involve head-on collisions with trains at grade crossings (Leibowitz, 1985). A report related to Brown's finding is that during visual tracking, the velocity of a large, moving grating pattern is typically underestimated, whereas the speed of a smaller grating pattern is not (Dichgans, Wist, Diener, \& Brandt, 1975). One of the intents of the present experiment was to systematically investigate the effect of target size on velocity perception during visual tracking while a constant extent and location of stationary surround contours were maintained.

In addition to target size, the presence of a background pattern has been found to produce misperceptions of target velocity during visual tracking. Raymond, Shapiro, and Rose (1984) investigated this phenomenon by having subjects visually track and judge the velocity of a small, smoothly moving target, which was viewed against a grating pattern that was drifting in either the same or the opposite direction as the target. They found that whereas target velocity was underestimated when the target was viewed against a background of stripes moving

This work was supported by a grant from NSERC. Thanks are extended to G. Silver for assistance in data collection and analysis and to an anonymous reviewer for helpful comments on an earlier draft. Requests for reprints should be directed to J. Raymond, Department of Psychology, University of Calgary, Calgary, AB T2N 1N4, Canada. in the same direction, it was overestimated if the same object was tracked against a grating drifting in the opposite direction.

The perception of object motion in this situation was probably derived from at least two sources: the efferent or corollary discharge signal related to the smooth eye movement used to track the target (Teuber, 1960) and the motion contrast produced at the edge of the target as it crossed the contoured background. Velocity misperception may result from an error in one or both signals. When velocity underestimation is experienced during the tracking of a large versus a small object viewed against a uniform field, the motion contrast produced at the edge of the target is eliminated as a factor, leaving the efferent signal associated with the smooth tracking eye movement as the most obvious source of error in the perception of object motion.

In the present experiment, the possibility of interactive effects of target size and background contour on velocity perception during tracking was investigated. The velocity perception of a smoothly moving target was assessed using a technique and stimuli similar to those described by Raymond et al. (1984). The target size and the direction of background motion were manipulated. The experiment was conducted using horizontal, upward, and downward motion.

\section{METHOD}

\section{Subjects}

Twenty university undergraduate students ( 3 male and 17 female) ranging in age from 16 to 28 years (mean $=20.9$ ) participated in the experiment. All subjects were naive as to the purpose of the experiment and were inexperienced psychophysical observers. Optical corrections appropriate for the viewing distance were worn as required during the experiment. All subjects had normal acuity and no history of visual or ocular disorder. 


\section{Apparatus}

A single display monitor (Tektronix 608, $\mathrm{P} 31$ phosphor) was used to generate both the moving target and the background stimuli. The display patterns were produced by a digital image generator (Picasso, Innisfree). The movement of the central square was controlled by a function generator (Wavetek 193). Both the function generator and the image generator were controlled by an appropriately interfaced microcomputer (Apple II+). The microcomputer timed stimulus presentation, produced auditory prompts, and recorded the subjects' responses.

The green, rectangular face of the monitor was surrounded by a large white screen that obscured the visibility of contours in the subjects' peripheral visual fields. The subjects viewed the display binocularly from a distance of $20 \mathrm{~cm}$ in a darkened room. A chinrest with a forehead restraining bar was used to stabilize head position.

\section{Stimuli}

The display consisted of a $31.8^{\circ} \times 26.6^{\circ}$ rectangular background and a smaller, moving target. The background was either a uniform field or a 0.44 -cycles/deg square-wave grating of $16 \%$ contrast. Contrast is defined as $\left(\mathrm{L}_{\max }-\mathrm{L}_{\min }\right) /\left(\mathrm{L}_{\max }+\mathrm{L}_{\min }\right) \times 100 \%$, where $\mathrm{L}_{\max }$ and $\mathrm{L}_{\min }$ refer to the maximum and minimum luminance on the screen, respectively. The spatial frequency and drift rate of the grating were chosen, on the basis of data from Schor and Narayan (1981), to provide optimal stimulation to the optokinetic system, since activation of this system might play a critical role in velocity misperception (see Discussion). A single bright or dark bar of the grating was narrower than the width of the smallest target. At all times during the experiment, the background had a space average luminance of $12.73 \mathrm{~cd} / \mathrm{m}^{2}$. Depending on the condition of the experiment, the grating was either horizontally or vertically oriented, and it drifted leftward, rightward, up, or down at a speed of $6.8 \mathrm{deg} / \mathrm{sec}$ (dps). When the orientation of the grating pattern was changed, the display monitor was rotated $90^{\circ}$ so that the movement of the target corresponded to the longer dimension of the rectangular screen in all conditions. The moving target was a square of four possible sizes $\left(1.43^{\circ}, 2.86^{\circ}, 5.71^{\circ}\right.$, or $\left.11.31^{\circ}\right)$ with a luminance of $5.43 \mathrm{~cd} / \mathrm{m}^{2}$. Movement of the target is described below.

\section{Procedure}

Each trial was composed of two target presentations. In the first presentation, a "standard" target appeared in one half of the screen and moved smoothly along the middle of the screen toward the other half at a constant velocity of $6.16 \mathrm{dps}$, and then it disappeared. Although the target always appeared initially in the same location, the distance over which it traveled varied randomly (as determined by the microcomputer) between $8.5^{\circ}$ and $14^{\circ}$. It was always viewed against a spatially uniform field and was always $1.43^{\circ}$ in size (i.e., the smallest target size).

After a 2-sec interval, the second ("test") target appeared at the same starting point and moved in the same direction along the same axis as the standard target. The test target was one of the four possible sizes described above, and it moved at one of five possible velocities $(3.91,4.93,6.16,7.80$, or $9.67 \mathrm{dps})$. The background against which the test target was viewed was either a spatially uniform field or a grating pattern that drifted with a constant velocity in either the same or the opposite direction as the target. Although the temporal frequency on the retina of the drifting grating varied for different target speeds, these temporal frequencies remained constant for different target sizes. As with the standard target, the distance over which the test target traveled varied randomly between $8.5^{\circ}$ and $14^{\circ}$, so that in either case, the duration of target presentation was not a reliable cue to target velocity. The subject's task on each trial was to judge whether the test target's velocity was faster or slower than that of the standard target. The subject signaled his/her choice by pressing the button on one of two appropri- ately labeled game paddles. The beginning of each trial was signaled by a tone. A different tone was used at the end of the trial as a prompt to respond.

Each experimental session consisted of 5 trials each of the 60 possible combinations of the three backgrounds, four target sizes, and five target speeds, for a total of $\mathbf{3 0 0}$ trials. These trials were broken up into four blocks of 75 trials. In the first and last blocks, the grating pattern, when present, moved in the direction opposite to that of the target. In the second and third blocks, the grating pattern drifted in the same direction as the target. Trials in which the test target was viewed against a uniform field were present in all blocks. Within a session, the direction of target movement and the orientation of the grating pattern were constant. Nine subjects performed the task with a rightward target motion and a vertically oriented grating pattern. Five different subjects performed the task with an upward target motion and a horizontally oriented grating pattern. An additional 6 subjects performed the task with a downward target motion and a horizontally oriented grating pattern.

The subjects began a session by reading written instructions, which strongly emphasized the need to track the moving targets with their eyes. ${ }^{1}$ In addition, the subjects were informed that the duration of target presentation would provide an unreliable cue to target velocity and that they should guess when they were unsure of velocity judgments. Each subject was given three practice trials. A 5-min rest break was given between each block of 75 trials. The session length was approximately $90 \mathrm{~min}$. The subjects were informed of the nature of the procedures, and their informed consent was obtained prior to their participation.

\section{RESULTS}

The present experiment demonstrates that both the size of a smoothly moving target and the background against which it is viewed affect perceived target velocity during ocular tracking. For each background condition, target size, and velocity, the percentage of trials on which each subject judged the test target to be moving faster than the standard target was determined and converted to a $z$-score. These values were plotted as a function of the log testtarget velocity for each background condition and target size. Least squares lines were calculated from each data set for each subject so that the resulting equation could be used to derive the test-target velocity, which corresponded to a $50 \%$ probability of responding " faster." This value, or velocity match, presumably reflects the velocity of the test target that the subject perceived to match that of the standard target. Velocity matches were averaged across subjects and are plotted as a function of target size in Figure 1.

In the figure, open circles represent the velocity matches obtained when the background was spatially uniform during test-target presentations. The closed squares indicate data obtained when a grating pattern drifted in the same direction as the test target, and the closed triangles indicate data obtained when a grating pattern drifted in the direction opposite to that of the test target. Standard-error bars (not shown) are just slightly larger than the symbols used. The dotted lines represent the actual velocity of the standard target. Thus, a point above the line indicates that the subjects perceived the test target and the standard target to be moving at the same speed, whereas the standard target was actually moving faster; they therefore underes- 

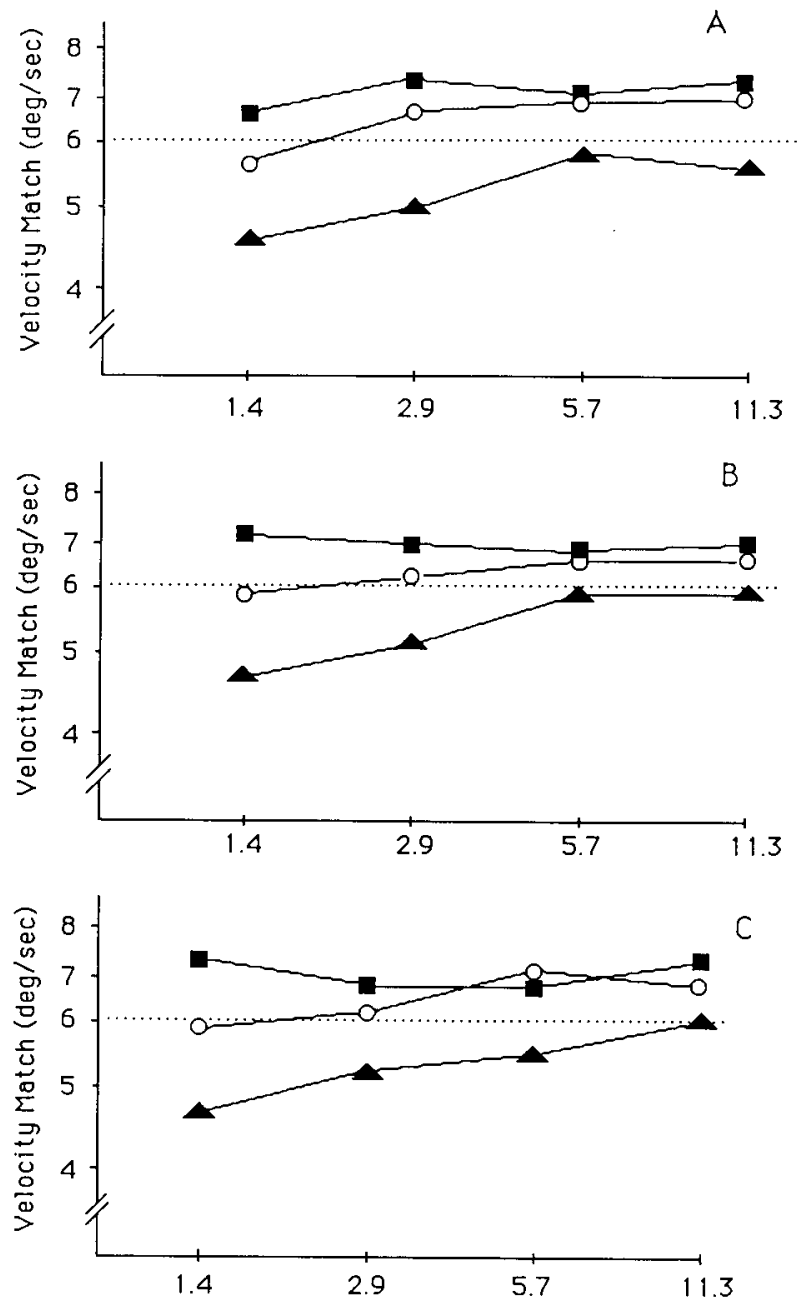

Figure 1. The group mean velocity matches plotted as a function of target size. Open circles represent data obtained when the background was spatially uniform throughout the trial. The filled squares and triangles represent data obtained when a striped background drifting with or against target motion, respectively, was present during the test target presentations. The dotted line represents the actual velocity of the standard target. Both axes are plotted on a $\log$ scale. A: The group mean velocity matches obtained when target motion was rightward. B: The group mean velocity matches obtained when target motion was upward. C: The group mean velocity matches obtained when target motion was downward.

timated the test target's velocity. Points below the line indicate an overestimation of target velocity. Panel A of Figure 1 illustrates the results obtained when target motion was rightward, and Panels $B$ and $C$ represent the data obtained when target motion was upward and downward, respectively.

An analysis of variance (ANOVA) on velocity matches for the rightward condition revealed a significant interaction effect of target size and background $[F(6,60)=$ $4.22, p<.001]$. Post hoc comparisons using Duncan's method were conducted. The effect of the background grating can be observed by examining the data points for the smallest target size. In these conditions, the standard and test targets were of the same size; therefore, any resulting over- or underestimation of test-target velocity must have been due to the presence of a background pattern. The results show that when the background grating drifted in the direction opposite to that of target motion, the velocity match values were significantly $(p<.01)$ slower (i.e., there was an overestimation of velocity) compared with the velocity match values derived for the uniform background condition. This difference was $0.09 \mathrm{log}$ units in size, and was equal to an overestimation of actual target velocity of $1.6 \mathrm{dps}$. The results show that when the background grating drifted in the same direction as the target, the velocity match values were significantly $(p<.01)$ faster (i.e., there was an underestimation of velocity) compared with the velocity match values derived for the uniform background condition. This difference was $0.06 \log$ units in size, and was equal to an underestimation of actual target velocity of $0.24 \mathrm{dps}$.

The effect of target size when the background remained spatially uniform can be seen by examining the open circles in the figure. As the size of the test targets increased, the test target velocities the subjects chose to match the standard target speed were faster than the ones they chose for the small test targets. This general effect was also observed when a grating pattern that moved in the direction opposite to target motion was present during the test-target presentation. For the uniform background conditions, this pattern of responses resulted in a mean velocity match value for the largest target that was significantly $(p<.01)$ faster than that for the smallest target. This difference in the velocity match values for the two extreme target sizes was $0.07 \mathrm{log}$ units, which represented a 1.2-fold change in perceived velocity for an 8-fold change in target size. Relative to actual target speed, the largest target's velocity was underestimated by $0.68 \mathrm{dps}$. For opposite-direction background motion conditions, an increase in target size acted to null the overestimation effect of the background. The velocity matches for the two larger target sizes were not significantly different from that obtained with the small target viewed against the uniform field, but were significantly different from that obtained with the smallest target viewed against oppositedirection backgrounds $(p<.01)$. When the grating moved in the same direction as the target, an increase in target size had little impact on the underestimation effect observed with the smallest target. Differences between the mean velocity matches for the largest and smallest target sizes were nonsignificant.

The results obtained with an ANOVA and post hoc comparisons performed on the data obtained in upward and downward conditions were the same as those obtained with the rightward-moving target. Significant interactions of background and target size were found for both the upward $[F(6,24)=6.46, p<.001]$ and downward $[F(6,30)$ $=3.42, p<.01]$ conditions. A similar pattern of significant differences between velocity match values was also found for both vertical conditions, and the magnitude of the effects was similar. 


\section{DISCUSSION}

\section{Effect of Target Size}

The results of the present experiment demonstrate that the size of a smoothly moving target affects perceived target velocity during ocular tracking. In the presence of a uniform background, an increase in target size produces a consistent, albeit modest, underestimation of target velocity. This is consistent with an early report by Brown (1931).

An obvious source of the perceptual error that leads to the observed velocity underestimation is the efference signal related to the smooth eye movement used to track the target. Recently, the role of efference in motion perception has been reevaluated in terms of the two oculomotor subsystems-one voluntary and one reflexive-that generate smooth tracking eye movements (Post, Schupert, \& Leibowitz, 1984; Raymond et al., 1984; Whiteside, Graybiel, \& Niven, 1965). Post and Leibowitz (1985) formalized these ideas into a theory that suggests that the efferent signal used in the perception of object motion is generated by activity of the voluntary tracking system and not by reflexively generated eye movements. This oculomotor theory of motion perception is based on the idea that both voluntary (i.e., smooth-pursuit) and reflexive (i.e., optokinetic or vestibulo-ocular) mechanisms may be stimulated simultaneously (Dichgans \& Brandt, 1978), and that a smooth tracking eye movement may be composed of both voluntary and reflexive components (Pola \& Wyatt, 1985). The passive viewing of large moving contours stimulates optokinetic eye movements (ter Braak, 1936), which are accompanied by the perception of selfmotion (Dichgans \& Brandt, 1978). Instructions to actively track a moving target produce voluntary smoothpursuit tracking, which is associated with the sensation of object motion. Voluntary pursuit is assumed to be the mechanism used to suppress reflexive eye movements when they are inappropriate (Dichgans \& Brandt, 1978; Schroeder, 1972; Troost, Dell'osso, \& Daroff, 1976), or to supplement them when they are insufficient to maintain gaze stability. If smooth-pursuit effort determines the magnitude of the efferent signal used in the perception of object motion, then the simultaneous contribution of both oculomotor systems to the output eye movement can be used to explain a wide variety of motion-perception illusions (e.g., Post, 1986). In general, this idea suggests that illusions of motion and misperceptions of target velocity should occur whenever both voluntary and reflexive eye-movement systems are simultaneously active in controlling the output eye movement.

Within the present context, the underestimation of target velocity that was observed with the larger targets may have resulted because the larger targets, which provided larger vertical contours, constituted a significant optokinetic stimulus. The stimulation of reflexive smooth tracking eye movements reduced the amount of voluntary smooth-pursuit effort required to track the target. Consequently, the efference copy associated with the volun- tary component of the eye movement that was used for object motion perception was lessened as stimulus size was increased, thus causing the underestimation of velocity.

Consistent with this interpretation is a recent report by Pola and Wyatt (1985). They demonstrated that when subjects were told to actively track moving targets, their eye movements were different for different sizes of the target. For the larger targets, the eye movements had characteristics (i.e., greater peak velocities and greater phase lags) that suggested that there was a component of optokinesis in the eye movement that was not present with the smaller targets. These data support the notion that during the tracking of the larger targets in the present experiment, the smooth tracking eye movements represented the combined output of both voluntary and reflexive mechanisms.

It is interesting to note, however, that the underestimation effect plateaued for relatively small stimuli $\left(5.7^{\circ}\right.$ square for upward and downward motion and $2.9^{\circ}$ square for rightward motion). Increasing the stimulus size up to $11.3^{\circ}$ square did not increase the magnitude of the underestimation effect. Schor and Narayan (1981) reported that sensitivity to stimuli that induce optokinetic nystagmus (OKN) was increased when stimulus field size was increased beyond $10^{\circ}$, which suggests that the effect of stimulus size on the underestimation effect observed here may be enhanced by targets larger than those used in the present study.

\section{Effect of Background}

When the subjects were required to make velocity judgments for the small targets in the presence of a background of stripes moving in the same direction as the target, an underestimation of target velocity was observed. An overestimation of target velocity was observed with a background of stripes moving in the direction opposite to that of the target. These results are consistent with a report by Raymond et al. (1984), in which a similar technique but slightly different stimuli were used.

As with the effect of target size, the effect of background may also be explained on the basis of differences in the magnitude of the efference copy associated with the voluntary component of the smooth tracking eye movement. When an observer tracks a target against a striped background that is moving in the direction opposite to that of the target, the optokinetic reflex to track the stripes must be suppressed, a function that is probably performed by voluntary tracking mechanisms (Dichgans \& Brandt, 1978; Schroeder et al., 1972; Troost et al., 1976). Thus, in this situation, voluntary smooth-pursuit effort is required both to track the target and to suppress the inappropriate optokinetic reflexive eye movements elicited by the moving background. The increased effort may then produce a larger efference copy, which may result in velocity overestimation. When the background and the target move in the same direction, reflexive and voluntary eye movements act synergistically. Since some of the 
tracking work is performed by the reflexive system, less effort is required from the voluntary smooth-pursuit system. The resulting smaller efference copy produces velocity underestimation.

An alternative explanation for the velocity under- and overestimation effects observed with drifting contoured backgrounds lies in the neural mechanisms that are sensitive to motion contrast. Frost and Nakayama (1983) described neural units in the pigeon optic tectum which could mediate motion illusions produced by motion contrast (e.g., induced motion). These cells responded maximally when a textured background pattern that stimulated the receptive field surround was moved in the direction opposite to that of a test spot that moved within the receptive field center of the cell. Inhibition was observed when the background moved in the same direction as the central spot. Such responsivity was not found when either the background or the test spot was presented alone, and it was largely independent of the axis of motion (i.e., vertical, horizontal, or oblique). Other neural mechanisms that may mediate relative motion perception have been described by Allman, Miezin, and McGuinness (1985) and others (Bridgeman, 1972; Burns, Gassanov, \& Webb, 1972; von Grünau \& Frost, 1983). In the Allman et al. study, the motion-sensitive units, which were recorded from the middle temporal lobe of the owl monkey and stimulated with a contour that moved in one direction within the classical receptive field, altered their response rates (in either an inhibitory or a facilitatory fashion, depending on the cell) when the visual field outside the classical receptive field was stimulated with motion in the opposite direction.

It has been suggested that activity of this class of neural mechanism mediates induced motion (Levi \& Schor, 1984). Since the stimuli and the task employed in the present study may be viewed as an induced-motion situation with a smooth tracking eye movement added, these neural mechanisms may also underlie the over- and underestimation effects observed here, thus eliminating the need to invoke an oculomotor explanation.

\section{Interaction of Background and Target Size}

When the background drifted against the target's motion, an increase in target size acted to null the velocity overestimation effect. Although motion-contrast neural mechanisms cannot easily account for the small effect of target size on velocity perception when there is no contour in the background, the activity of these mechanisms may explain the interactive effect of background and target size. If, for the small targets, the activation of the motion-contrast-sensitive units produced errors in velocity perception, as they may do when the eye is stationary in an induced-motion situation, then an increase in target size may act to decrease their responsivity by stimulating parts of the motion-antagonistic receptive field surround.

An alternate explanation lies in the optokinetic suppression idea of Post and Leibowitz (1985). If the larger target provided a more optimal stimulus for optokinetic eye movements in the direction of the target, and if the back- ground provided an optokinetic stimulus in the direction of the background, then, because they act in opposite directions, the two signals would cancel. The efference copy associated with the tracking of the large target against this background would be the same as the tracking of the small target against a uniform background, since in both cases there is virtually no effective optokinetic stimulation present. When the background grating and the target are moving in the same direction, any optokinetic properties of the background or the target work together to elicit a reflexive eye movement in the same direction as target motion.

\section{Effect of Direction of Background Drift}

In general, there was a notable lack of difference in the velocity perception effects observed with vertically versus horizontally moving backgrounds (and targets) and for upward versus downward motion of the target. Some researchers have reported differences in optokinetic responses that depend on the direction of motion of the OKN-inducing stimulus (Hulls \& Stockwell, 1974; Matsuo, Cohen, Raphan, de Jong, \& Henn, 1979). To examine this possibility within the present context, an experiment using 2 observers was conducted in which OKN was measured (using an infrared corneal-reflection eyemovement monitoring device) in response to the backgrounds used in the perception experiments. To be certain that the eye movements represented OKN rather than voluntary pursuit tracking (Cheng \& Outerbridge, 1974), only OKNs with a fast-phase frequency equal to or greater than $1 \mathrm{~Hz}$ were used in the analysis. The results showed a slightly lower slow-phase gain for vertical OKNs (mean $=0.83$ ) than for horizontal (mean $=1.01)$, and no difference between upward and downward contour motion. If slow-phase gain is an indication of the effectiveness of an optokinetic stimulus, the present results suggest that the degree of optokinetic effectiveness of the background (within the range used here) did not influence the velocityperception effects induced by it.

The present experiment has demonstrated that the perceived velocity of a visually tracked target depends not only on the size of the target, but also on the presence of contours in the visual background. Within the range of target sizes studied here, the data suggest that, in the natural environment in which richly contoured backgrounds are typically available, subtle overestimation of the velocity of small targets may be experienced and underestimation of the velocity of large targets is not likely to occur.

\section{REFERENCES}

Allman, J., Miezin, F., \& McGuinness, E. (1985). Direction- and velocity-specific responses from beyond the classical receptive field in the middle temporal visual area (MT). Perception, 14, 105-126.

BRIDGEMAN, B. (1972). Visual receptive fields sensitive to absolute and relative motion during tracking. Science, 178, 1106-1108.

Brown, J. F. (1931). The visual perception of velocity. Psychologische Forschung, 14, 199-232.

Burns, B. D., Gassanov, U., \& WebB, A. C. (1972). Response of 
neurons in the cat's visual cerebral cortex to relative movement of patterns. Journal of Physiology, 276, 133-151.

Cheng, M., \& OUTERBRIDGE, J. S. (1974). Inter-saccadic interval analysis of optokinetic nystagmus. Vision Research, 14, 1053-1058.

Dichgans, J., \& BRANDT, T. (1978). Visual-vestibular interaction: Effects on self-motion perception and postural control. In R. Held, H. W. Leibowitz, \& H.-L. Teuber (Eds.), Handbook of sensory physiology (Vol. 8, pp. 755-807). Berlin: Springer.

Dichgans, J., Wist, E., Diener, H. C., \& Brandt, T. (1975). The Aubert-Fleischl phenomena: A temporal frequency effect on perceived velocity in afferent motion perception. Experimental Brain Research, 23, 529-533.

Frost, B. J., \& NaKayama, K. (1983). Single visual neurons code opposing motion independent of direction. Science, 220, 744-745.

Hulls, J. R., \& STockwell, C. W. (1974). Suppression of optokinetic nystagmus. Minerva Otorinolaringologica, 24, 261-265.

LEIBowITZ, H. W. (1985). Grade crossing accidents and human factors engineering. American Scientist, 73. 558-562.

Levi, D., \& SCHOR, C. (1984). Spatial and velocity tuning of processes underlying induced motion. Vision Research, 24, 1189-1196.

Matsuo, V., Cohen, B., Raphan, T., de Jong, V., \& Henn, V. (1979). Asymmetric velocity storage for upward and downward nystagmus. Brain Research, 176, 159-164.

Pola, J., \& WyatT, H. J. (1985). Active and passive smooth eye movements: Effects of stimulus size and location. Vision Research, 25, 1063-1076.

Post, R. B. (1986). Induced motion considered as a visually induced oculogyral illusion. Perception, 15, 131-138.

Post, R. B., \& LeIBowtTz, H. W. (1985). A revised analysis of the role of efference in motion perception. Perception, 14, 631-645.

Post, R. B., Schupert, C. L., \& Leibowitz, H. W. (1984). Implications of OKN suppression by smooth pursuit for induced motion. Perception \& Psychophysics, 36, 493-498.

Raymond, J. E., Shapiro, K. L., \& Rose, D. J. (1984). Optokinetic backgrounds affect perceived velocity during ocular tracking. Perception \& Psychophysics, 36, 221-224.

Schor, C., Narayan, V. (1981). The influence of field size upon the spatial frequency response of optokinetic nystagmus. Vision Research, 21, 985-994.

SCHRoEder, D. J. (1972). Some effects of alcohol on nystagmus and vertigo during caloric and optokinetic stimulation. Annals of Otol ogy, Rhinology, \& Laryngology, 81, 218-229.

TER BRAAK, J. W. C. (1981). Untersuchungen über optokinetischen Nystagmus (H. Colleweijn, Trans.). In Oculomotor system of the rabbit and its plasticity. New York: Springer-Verlag. (From Archives Neerlandaises de Physiologie de lhomme et des Animaux, 1936, 21, 309-376)

Teuber, H.-L. (1960). Perception. In H. Magoun (Ed.), Handbook of physiology: III. Section of neurophysiology (pp. 1595-1688). Washington, DC: American Physiological Society.

Troost, B. T., Dellosso, L. F., \& DarofF, R. B. (1976). Effects of visual pursuit deficit on the human vestibulo-ocular reflex. Neurology, 26, 352-353.

von Grünau, M., \& Frost, B. J. (1983). Double-opponent-process mechanism underlying RF-structure of directionally specific cells of cat lateral suprasylvian visual area. Experimental Brain Research, 49, 84-92.

Whiteside, T. D. C., Graybiel, A., \& Niven, J. I. (1965). Visual illusions of movement. Brain, 88, 193-210.

\section{NOTE}

1. Previous research (Raymond et al., 1984) using this procedure demonstrated that instructions to fixate a stationary point during the task and instructions to track the target produced different results, which was supportive of the contention that subjects behaved as instructed. In the present study, the monitoring of eye movement during the velocitymatching task described above demonstrated that the subjects did use smooth-pursuit tracking to follow the target on each presentation.

(Manuscript received October 27, 1986; revision accepted for publication September 29, 1987.) 